\author{
И. П. Деревяго ${ }^{1}$, Д. Г. Малашевич ${ }^{2}$ \\ ${ }^{1}$ Белорусский государственный университет \\ ${ }^{2}$ Белорусский государственный технологический университет
}

\title{
ЦИРКУЛЯРНАЯ ЭКОНОМИКА КАК ИНСТРУМЕНТ ЗЕЛЕНОГО РОСТА
}

Статья посвящена проблемам развития циркулярной экономики и формирования соответствующей экономической политики. На основе анализа литературы был сделан вывод об отсутствии единой концепции циркулярной экономики, которая скорее рассматривается как инструмент, чем как самостоятельная область исследований и практической деятельности. В зависимости от того, для достижения каких целей используется данная концепция, будет зависеть ее содержание. С учетом характера современной политики в области устойчивого развития было принято рассматривать циркулярную экономику как инструмент зеленого роста. Анализируя в данном контексте политику ее развития, целесообразно ориентироваться на ключевые требования последнего. В работе предлагается осуществлять развитие циркулярной экономики в рамках политики зеленого роста, с выделением пяти основных компонентов: институционального регулирования, регулирования спроса, регулирования предложения, финансирования, информационного обеспечения. Базируясь на международном опыте и особенностях развития белорусской экономики для каждого из пяти компонентов, авторы предлагают наиболее актуальные направления, которые целесообразно отразить и раскрыть в процессе развития циркулярной экономики. С учетом того, что государственный сектор в нашей стране занимает доминирующее положение, одним из ключевых факторов успеха в данном случае рассматривается активная роль государства.

Ключевые слова: циркулярная экономика, отходы, зеленый рост, экономическая политика, финансирование, информационное обеспечение.

Для цитирования: Деревяго И. П., Малашевич Д. Г. Циркулярная экономика как инструмент зеленого роста // Труды БГТУ. Сер. 5, Экономика и управление. 2021. № 1 (244). С. 97-102.

\author{
I. P. Dzeraviaha ${ }^{1}$, D. G. Malashevich ${ }^{2}$ \\ ${ }^{1}$ Belarusian State University \\ ${ }^{2}$ Belarusian State Technological University
}

\section{CIRCULAR ECONOMY AS A TOOL FOR GREEN GROWTH}

The article is devoted to the problems of the development of a circular economy and the formation of an appropriate economic policy. Based on the analysis of the literature, it was concluded that there is no unified concept of circular economy, which is more likely seen as a tool than as an independent area of research and practice. Depending on what goals this concept is used to achieve, its content will depend. Given the nature of modern sustainable development policies, it has been accepted to view the circular economy as a tool for green growth. Analyzing its development policy in this context it is advisable to focus on the key requirements of the latter. The paper proposes to develop a circular economy within the framework of a green growth policy, highlighting five main components: institutional regulation, demand regulation, supply regulation, financing, information support. Based on international experience and the peculiarities of the development of the Belarusian economy, for each of the five components, the most relevant directions are proposed, which are advisable to reflect and disclose in the process of developing a circular economy. Given that the public sector in our country occupies a dominant position, one of the key success factors in this case is considered the active role of the state.

Key words: circular economy, waste, green growth, economic policy, financing, information support.

For citation: Dzeraviaha I. P., Malashevich D. G. Circular economy as a tool for green growth. Proccedings of BSTU, issue 5, Economics and Management, 2021, no. 1 (244), pp. 97-102 (In Russian).

Введение. Необходимость перехода к устойчивому развитию и пересмотра ориентированной на максимизацию потребления экономической парадигмы привела к появлению ряда междисциплинарных подходов, направленных на интегрированный анализ экономических и экологических процессов. Одним из таких подходов является циркулярная экономика, которая 
предполагает переход производства и потребления от линейной модели функционирования к циркулярной.

Впервые циркулярную экономику в качестве самостоятельного направления исследования определили Д. Пирс и Р. Тернер более 30 лет назад [1].

С тех пор существенно возросла заинтересованность в ее развитии со стороны как ученых, так и практиков. Высокую востребованность циркулярной экономики подтверждает внимание, которое ей уделяется в политике ряда стран. В частности, план внедрения циркулярной экономики занимает одно из центральных мест в рамках амбициозной программы ЕС по развитию зеленой экономики (Green deal), которая предполагает более 500 млрд евро инвестиций экономики до 2050 г.

Тем не менее до настоящего времени отмечается достаточно широкий разброс мнений относительного содержания и целей циркулярной экономики, что усложняет ее практическую реализацию. На основе анализа существующих концепций циркулярной экономики в статье предложен подход к ее определению в качестве инструмента зеленого роста, с учетом чего рассмотрены наиболее важные направления практической реализации.

Основная часть. Анализ научной и методической литературы показывает, что понятие циркулярной экономики трактуется достаточно широко. Многообразие подходов демонстрируют исследования, которые позволяют выделить 72 концепции [1] и 114 определений [2] циркулярной экономики. Разные исследователи акцентируют внимание на самых различных аспектах [3]. Большая группа специалистов подчеркивает тесную взаимосвязь циркулярной экономики с устойчивым развитием. Для многих практиков центральным элементом исследований является техническая сторона, связанная с зацикливанием материальных потоков. Ряд экологов отмечают в качестве первоочередной задачи циркулярной экономики - управление отходами и снижение воздействия на окружающую среду. Важное внимание уделяется экоинновациям и факторам, которые способствуют их разработке и распространению [4]. Подобные подходы отражают значительный технологический уклон циркулярной экономики. В то же время, несмотря на свое название, данная концепция не находит широкого применения в экономической науке [5].

Учитывая многообразие подходов к циркулярной экономике, сложно сформулировать определение, способное объединить их в полной мере и отразить данное многообразие. Не останавливаясь на детальном анализе, нужно отметить, что выработка консенсуса в отношении содержания и структуры циркулярной экономики требует конкретизации основных атрибутов, которые характеризуют любую область исследования, - методологии, предмета и целей.

Выбор метода (системы методов или методологии) является важнейшим фактором, от которого зависят полнота и достоверность полученных результатов, эффективность построенных моделей, степень достижения поставленной цели. Характерной чертой циркулярной экономики является ее направленность на конкретную методологию. Именно ориентированность на замкнутый цикл в противоположность линейной модели производства и потребления стала причиной возникновения данной концепции. Исходя из названия, циркулярная экономика не столько ориентирована на конкретный предмет, сколько на методологию, которая подразумевает переход от линейной к циркулярной модели производства и потребления. Выражением методологии циркулярной экономики можно считать R-концепцию. Наиболее часто упоминается 3R-концепция (от английского reduce (снизить), reuse (заново использовать), recycle (вернуть в оборот)). В более широкой трактовке R-концепция может быть расширена до 5,7 и $10 \mathrm{R}$ [3].

Вместе с тем одной методологии недостаточно, чтобы рассматривать циркулярную экономику как самостоятельное направление. Цикличность либо создание замкнутого цикла не могут быть самоцелью. Во-первых, согласно законам термодинамики, абсолютно замкнутый цикл невозможен. А во-вторых, стремление к максимальной замкнутости цикла в рамках одного объекта может стать причиной дополнительного воздействия на окружающую среду за его пределами и привести к снижению экологической устойчивости в целом [6]. Поэтому важно четко определить сферу применения и цели циркулярной экономики, которые существенно менялись по мере ее эволиции. В историческом контексте можно выделить следующие четыре концепции циркулярной экономики [1]:

1) циркулярная экономика как модель управления отходами. Это наиболее узкий подход, направленный на решение проблемы переработки отходов. Его возникновение стало реакцией на рост загрязнения и обусловило зарождение циркулярной экономики;

2) ииркулярная экономика как способ повышения ресурсоэффективности. В рамках этой концепции основной целью циркулярной экономики 
является внедрение технологий и продуктов, которые позволяют обеспечить замкнутый цикл материальных потоков. Она отражает технологический подход, который в большей степени соотносится с промышленной экологией, экодизайном и пр. На сегодняшний день данная концепция остается наиболее востребованной на практике;

3) циркулярная экономика как способ организачии экономической деятельности в целом. Этот подход выходит за технологические рамки, поскольку рассматривается циркулярная экономика как модель производства и потребления, ориентированная на достижение экономического роста при соблюдении требований экологической устойчивости (зеленого роста). В отличие от предыдущей, данная концепция ориентирована не только на технические решения, но и на институциональные изменения, формирование новых моделей поведения потребителей;

4) циркулярная экономика как альтернатива современной сочиально-экономической модели. Это наиболее радикальный подход к циркулярной экономике, в рамках которого экономический рост не является приоритетом. Основной целью циркулярной экономики в таком случае выступает необходимость полной гармонизации общества и окружающей среды, тогда как проблема экономического роста снимается с повестки дня.

С учетом современных приоритетов в экономической политике как на международном уровне, так и на уровне отдельных государств наиболее адекватной является третья концепция. Ее содержание в значительной степени соответствует позиции стран Европейского союза, Японии, других государств, которые ориентируются на применение широкого круга мер по обеспечению экономического роста за счет повышения ресурсоэффективности. Наглядным примером демонстрации подхода, в рамках которого циркулярная экономика рассматривается как инструмент зеленого роста, является План действий по ее развитию в Европейском Союзе (Circular Economy Action Plan ${ }^{1}$ ). Он выступает составным компонентом так называемой «зеленой инициативы или сделки» (European Green Deal) и охватывает следующие области:

- политику по внедрению модели устойчивого производства и потребления, включая совершенствование дизайна продуктов, расширение возможностей потребителей по их более длительному и безопасному использованию, стимулирование повторного использования производственных отходов;
- цепочки ценности для ключевых продуктов, в том числе электроники, компьютеров, телефонов, транспортных средств и аккумуляторных батарей, упаковки и изделий из пластика, текстиля, строительных материалов, продуктов питания;

- минимизация отходов и повышение ценности от использования материальных ресурсов за счет мер по предотвращению отходов, снижению их токсичности, созданию эффективных рынков вторичных материальных ресурсов;

- создание рабочих мест, формирование циркулярной экономики, которая будет способствовать развитию городов и регионов;

- интеграция циркулярной экономики в процесс устойчивого экономического развития экономики в целом, повышение ее роли в решении проблемы климатических изменений, достижение максимального эффекта за счет цифровизации, совершенствования налогообложения и пр.

В последние годы все больше стран и компаний осознает необходимость соответствия принципам циркулярной экономики. При этом их внедрение рассматривается не как самоцель, а как компонент более широкой инициативы по достижению целей устойчивого развития и переходу к зеленому росту, что подтверждает пример Европейского союза. С учетом данного подхода и целесообразно формировать целевые ориентиры для циркулярной экономики. В свою очередь, исходя из содержания политики зеленого роста [7], можно выделить следующие ключевые для ее развития аспекты:

- институциональное регулирование;

- регулирование спроса;

- регулирование предложения;

- финансирование;

- информационное обеспечение.

Институциональное регулирование. Институты определяют поведение потребителей и производителей, поэтому институциональное регулирование может рассматриваться в качестве ключевого фактора при формировании модели циркулярной экономики. Понятие институтов достаточно широко, институциональные меры затрагивают и регулирование спроса, и регулирование предложения. Тем не менее выделение институционального компонента важно, поскольку с его помощью формируются рамки, в которых принимаются решения участниками экономических отношений. В данном контексте необходимо отметить разделение институтов на неформальные и формальные. Первые обусловлены

\footnotetext{
${ }^{1}$ Communication from the commission to the European parliament, the council, the European economic and social committee and the committee of the regions. https://eurlex.europa.eu/resource.html?uri=cellar:9903b325-6388-11ea-b735-01aa75ed71a1.0017.02/DOC_1\&format=PDF
} 
сложившимися традициями, привычками потребления и производственным поведением. Часто, что особенно характерно для Беларуси, ресурсосбережение может быть финансово выгодно, однако компании не предпринимают шагов в данном направлении по причине сложившейся культуры управления, отсутствия стимулов у руководителей и исполнителей, по другим причинам. В этом случае можно говорить об неэффективности институтов в целом, безотносительно к циркулярной экономике. А стратегия развития последней должна быть направлена на институциональные улучшения в целом, включая распространение экологически ориентированной культуры управления, потребления, производства.

Формальные институты, как правило, определятся законодательными и нормативными документами. С точки зрения циркулярной экономики формальное институциональное регулирование (налоги, экологические нормы, зеленые тарифы и пр.) имеет важное значение, поскольку в значительной степени влияет на конкурентоспособность циркулярных моделей и возможность достижения приемлемого уровня экономической эффективности.

Регулирование спроса. Традиционным индикатором экономического роста является изменение ВВП. Рассматривая циркулярную экономику как инструмент зеленого роста, регулирование спроса целесообразно исследовать в разрезе компонентов совокупного спроса, которые включает ВВП, - государственный, потребительский, корпоративный и внешний сектора.

Формирование спроса со стороны государства происходит как напрямую, за счет расходов государственного бюджета и закупок государственных учреждений, так и косвенно, через спрос организаций и предприятий, которые контролируются государством. Важными инструментами регулирования спроса в данном случае выступают зеленые закупки, бюджетные субсидии и т.п. С учетом значительной роли государства в белорусской экономике именно спрос со стороны государственного сектора может стать ключевым условием развития циркулярной экономики.

При регулировании спроса потребительского и корпоративного сектора в целях развития циркулярной экономики целесообразно различать ценовые и неценовые факторы. Главным инструментом воздействия на цены являются налоги на потребление, которые, как показывает практика, могут существенно влиять на потребление продуктов в условиях высокой эластичности спроса по цене. Что касается неценовых факторов, то они включают в себя широкий набор мер, в частности экологический маркетинг и сертификацию, информирование, стандарты обслуживания потребителей, формирование соответствующей потребительской психологии и пр.

Регулирование предложения. Политика регулирования предложения ориентирована на разработку, совершенствование и повышение доступности «циркулярных» технологий. Систему мер по регулированию предложения можно представить иерархией:

1) национальный уровень: формирование экологически ориентированной отраслевой структуры экономики. Особенно это касается инвестиционной политики. Отраслевая структура инвестиций определяет технологический профиль экономики на годы вперед, формируя, таким образом, ресурсоемкость и ресурсоэффективность производств, уровень их экологической опасности;

2) отраслевой уровень: адаптация и масштабирование циркулярных бизнес-моделей (инноваций) в рамках технологически однородных видов деятельности;

3) уровень производителя: интенсификация инноваций в области циркулярной экономики и ресурсосбережения. На данном уровне важную роль играют создание соответствующей финансовой и информационной инфраструктуры.

Финансирование. Политика финансирования циркулярной экономики должна учитывать два основных аспекта:

- соответствие инвестиционной политики государства принципам циркулярной экономики в целом. Повышение инвестиционной привлекательности трудоемких и экологически безопасных видов деятельности позволит существенно повысить ресурсоэффективность и ускорить внедрение принципов циркулярной экономики;

- возможности финансирования циркулярных проектов. Учитывая, что такие проекты часто являются рискованными и не всегда коммерчески привлекательными, важно рассмотреть возможность использования не только традиционных источников инвестиций, но и специальных инструментов, включая зеленые облигации, привлечение средств международных фондов и организаций; зеленые венчурные фонды и пр.

Информаџионное обеспечение. Реализация эффективной политики предполагает наличие информации для принятия решений и четких критериев для оценки их эффективности. Анализ показал, что в Беларуси в последние годы 
отмечается существенное улучшение информационного обеспечения в области зеленого роста и циркулярной экономики [8].

Тем не менее ряд важных для оценки ресурсоэффективности показателей пока в нашей стране официально не учитывается. К таким показателям можно отнести внутреннее потребление материальных ресурсов (domestic material consumption), уровень циркулярности использования ресурсов (circular material use rate) и др. Улучшение информационного обеспечения позволит значительно повысить эффективность политики в области циркулярной экономики и зеленого рост в целом.

Заключение. Для реализации эффективной политики в области циркулярной экономики последнюю целесообразно рассматривать как ин- струмент зеленого роста. Это позволяет выделить пять основных компонентов в системе регулирования циркулярной экономики: институты, спрос, предложение, финансирование и информационное обеспечение. В рамках перечисленных направлений важно обеспечить поэтапную реализацию соответствующей политики, включая проведение институциональных преобразований, определение приоритетных направлений развития циркулярной экономики, совершенствование информационного обеспечения, создание необходимой инфраструктуры. Одним из ключевых факторов успеха развития циркулярной экономики является активная роль государства. Особенно это касается белорусской экономики, в которой государственный сектор занимает доминирующее положение.

\section{Список литературы}

1. Friant M. C., Vermeulen W., SalomoneR.. A typology of circular economy discourses: Navigating the diverse visions of a contested paradigm // Resources, Conservation and Recycling. 2020. Vol. 161. Available at: https://doi.org/10.1016/j.resconrec.2020.104917/ (accessed 12.01.2021).

2. Kirchherr J., Reike D., Hekkert M. Conceptualizing the circular economy: An analysis of 114 definitions // Resources, Conservation and Recycling. 2017. Vol. 127. P. 221-232.

3. Reike D., Vermeulen, W., Witjes S. The circular economy: new or refurbished as CE 3.0? - exploring controversies in the conceptualization of the circular economy through a focus on history and resource value retention options // Resources, conservation and recycling. 2018. Vol. 137. P. 246-264.

4. Prieto-Sandoval V., Jaca C., Ormazabal M. Towards a consensus on the circular economy // Journal of Cleaner Production. 2018. Vol. 179. P. 605-615.

5. Velis C. No circular economy if current systemic failures are not addressed // Waste Management \& Research. 2018. Vol. 36 (9). P. 757-759.

6. Blum N., Haupt M., Bening C. Why "Circular" doesn't always mean "Sustainable" // Resources, Conservation and Recycling. 2020. Vol. 162. Available at: https://doi.org/10.1016/j.resconrec.2020.105042/ (accessed 12.02.2021).

7. Деревяго И. П. Условия и возможности перехода экономики к зеленому росту // Белорусский экономический журнал. 2020. № 4. С. 20-35.

8. Деревяго И. П., Малашевич Д. Г. Проблемы совершенствования оценочной системы зеленого роста в Республике Беларусь // Труды БГТУ. Сер. 5, Экономика и управление. 2020. № 1 (232). C. $85-89$.

\section{Reverences}

1. Friant M. C., Vermeulen W., SalomoneR.. A typology of circular economy discourses: Navigating the diverse visions of a contested paradigm. Resources, Conservation and Recycling, 2020, vol. 161. Available at: https://doi.org/10.1016/j.resconrec.2020.104917/ (accessed 12.01.2021).

2. Kirchherr J., Reike D., Hekkert M. Conceptualizing the circular economy: An analysis of 114 definitions. Resources, Conservation and Recycling, 2017, vol. 127, pp. 221-232.

3. Reike D., Vermeulen, W., Witjes S. The circular economy: new or refurbished as CE 3.0? - exploring controversies in the conceptualization of the circular economy through a focus on history and resource value retention options. Resources, conservation and recycling, 2018, vol. 137, pp. 246-264.

4. Prieto-Sandoval V., Jaca C., Ormazabal M. Towards a consensus on the circular economy. Journal of Cleaner Production, 2018, vol. 179, pp. 605-615.

5. Velis C. No circular economy if current systemic failures are not addressed. Waste Management \& Research, 2018, vol. 36 (9), pp. 757-759.

6. Blum N., Haupt M., Bening C. Why "Circular" doesn't always mean "Sustainable". Resources, Conservation and Recycling, 2020, vol. 162. Available at: https://doi.org/10.1016/j.resconrec.2020.105042/ (accessed 12.02.2021). 
7. Derevyago I. P. Conditions and opportunities for the transition to green growth. Belorusskiy ekonomicheckiy zhurnal [Belarusian Economic Journal], 2020, no. 4, pp. 20-35 (In Russian).

8. Derevyago I. P., Malashevich D. G. Problems of improving the green growth appraisal system in the Republic of Belarus. Trudy BGTU [Proceedings of BSTU], issue 5, Economics and Management, 2020, no. 1 (232), pp. 85-89 (In Russian).

\section{Информация об авторах}

Деревяго Игорь Петрович - кандидат экономических наук, доцент, заведующий кафедрой корпоративных финансов. Белорусский государственный университет (220004, г. Минск, ул. Обойная, 7, Республика Беларусь). E-mail: Dzeraviaha@sbmt.by

Малашевич Диана Георгиевна - старший преподаватель кафедры менеджмента, технологий бизнеса и устойчивого развития. Белорусский государственный технологический университет (220006, г. Минск, ул. Свердлова, 13а, Республика Беларусь). E-mail: malashevich@belstu.by

\section{Information about the authors}

Dereviaha Ihar Petrovich - PhD (Economics), Associate Professor, Head of the Department of Corporate Finance. Belarusian State University (7, Oboynaya str., 220004, Minsk, Republic of Belarus). E-mail: Dzeraviaha@sbmt.by

Malashevich Diana Georgievna - Senior Lecturer, the Department of Management, Business Technology and Sustainable Development. Belarusian State Technological University (13a, Sverdlova str., 220006, Minsk, Republic of Belarus). E-mail: malashevich@belstu.by 